\title{
Dependence on the environment of the abundance function of light-cone simulation dark matter haloes
}

\author{
Maria Chira $^{1,2}$, Manolis Plionis ${ }^{1,2}$, and Pier-Stefano Corasaniti ${ }^{3}$ \\ 1 Physics Department, Aristotle University of Thessaloniki, Thessaloniki 54124, Greece \\ e-mail: mchira@physics .auth.gr \\ 2 National Observatory of Athens, Lofos Nymfon, 11852 Athens, Greece \\ 3 LUTH, UMR 8102 CNRS, Observatoire de Paris, PSL Research University, Université Paris Diderot, 92190 Meudon, France
}

Received 24 June 2017 / Accepted 16 May 2018

\begin{abstract}
Aims. We study the dependence of the halo abundance function (AF) on different environments in a whole-sky $\Lambda \mathrm{CDM}$ light-cone halo catalogue extending to $z \sim 0.65$, using a simple and well-defined halo isolation criterion.

Methods. The isolation status of each individual dark matter halo is determined by the distance to its nearest neighbour, which defines the maximum spherical region devoid of halos above a threshold mass around it (although the true size of such region may be much larger since it is not necessarily spherical). A versatile double power-law Schechter function is used to fit the dark matter halo AF, and its derived parameters are studied as a function of halo isolation status.

Results. (a) Our function fits the halo abundances for all halo isolation statuses extremely well, while the well-established theoretical mass functions, integrated over the volume of the light-cone, provide an adequate but poorer fit than our phenomenological model. (b) As expected, and in agreement with other studies based on snap-shot simulations, we find significant differences of the halo abundance function as a function of halo isolation, indicating different rates of halo formation. The slope of the power law and the characteristic mass of the Schechter-like fitting function decrease with isolation, a result consistent with the formation of less massive haloes in lower density regions. (c) We find an unexpected upturn of the characteristic mass of the most isolated haloes of our sample. This upturn originates and characterises only the higher redshift regime $(z \gtrsim 0.45)$, which probably implies a significant and recent evolution of the isolation status of the most isolated and most massive haloes.
\end{abstract}

Key words. methods: numerical - large-scale structure of Universe - methods: statistical - dark matter

\section{Introduction}

According to the cold dark matter (DM) paradigm, cosmic structures form hierarchically as a result of the growth of primordial density perturbations. The resulting fundamental non-linear cosmic structures are known as dark matter haloes, in the interior of which baryonic matter collapses to form galaxies, groups of galaxies and clusters of galaxies.

It is well established that the distribution of cosmic structures is far from uniform. DM haloes, and consequently, the visible objects they host, form a hierarchy of cosmic structures from pairs to superclusters of galaxies, constituting what has been called the "cosmic web" by Bond et al. (1996), which has revealed a wealth of different environments. A fundamental property that has emerged from observations as well as from $\mathrm{N}$-body simulations, and which is environmental in its essence, is that they are more clustered in comparison to the underlying mass fluctuations. This property is called bias and is explained as being the result of structures forming at the peaks of the initial random Gaussian density field (e.g. Kaiser 1984; Peacock \& Heavens 1985).

The importance of the cosmic environment was first provided through indications for an environmental dependence of galaxy properties by Dressler (1980), who showed that galaxy Hubble type and ambient galaxy density are tightly correlated. Since the unveiling of the cosmic web, interest has been growing, and systematic studies have been conducted to quantiyfy and explain the environmental dependencies of galaxy properties.

Recent studies have shown that the environment correlates not only with various galactic properties (e.g. Gómez et al. 2003; Boselli \& Gavazzi 2006; Blanton \& Berlind 2007; Croton et al. 2007; Forero-Romero et al. 2011; Eardley et al. 2015; Metuki et al. 2015), but also with the properties of the DM halo within which they reside (e.g. Navarro et al. 1997; Bullock et al. 2001; Schuecker et al. 2001; Plionis \& Basilakos 2002; Wechsler et al. 2002, 2006; Gao et al. 2004, 2005; Sheth \& Tormen 2004; Avila-Reese et al. 2005; Harker et al. 2006; Martínez \& Muriel 2006; Zhu et al. 2006; Gao \& White 2007; Ragone-Figueroa \& Plionis 2007; Libeskind et al. 2011, 2012, 2013, 2014; Lee et al. 2017). This indicates that quantifying the inter-relation between halo properties (e.g. shapes, accretion rates, spin parameters, alignments, substructure, and formation times) and the environment, local and large scale, can shed light onto the structure formation processes.

A crucial point that has emerged in all studies is the definition of the environment itself. A large variety of methods has been used to quantify the effect of the environment on the distribution of galaxies and DM haloes (for an overview, see Muldrew et al. 2012; Libeskind et al. 2018). As an example, some of the works have used a nearest-neighbour approach, while others have defined the ambient density field after applying a variety of smoothing kernels to the point-like distribution of haloes. 
One of the many properties of the galaxy and halo distributions that seems to correlate with the environment is the halo mass function, the study of which is crucial in order to develop an understanding of galaxy and structure formation processes. Pioneering work on these issues is that of Press \& Schechter (1974), extended by Bond et al. (1991) to include the excursion set formalism and by Sheth et al. (2001) to include the more realistic ellipsoidal collapse model which takes into account the triaxiality of the Gaussian density field perturbations (Doroshkevich 1970; Bardeen et al. 1986). The resulting DM halo mass functions were further improved by many other studies (e.g. Sheth \& Tormen 1999; Jenkins et al. 2001; Warren et al. 2006; Reed et al. 2007; Valageas 2009; Tinker et al. 2010; Ma et al. 2011; Corasaniti \& Achitouv 2011).

Furthermore, other possible environmental dependencies of the halo and galaxy properties could be an ingredient of structure formation processes and thus many of the studies cited previously have investigated such dependencies. A quite common result of such studies is the higher abundance of massive haloes in dense environments (e.g. Lemson \& Kauffmann 1999; Maulbetsch et al. 2007)

Thus the abundance of haloes of different mass seems to differ in different environments. However, it is not yet clear if this effect depends on the ambient density or on the web-element type (knots, filaments, sheets, or voids). Hahn et al. (2007) reported a variation in halo mass function with web-element classification, in the direction of an increasing higher mass end of the halo mass function at the upper part of the web-element sequence, which corresponds to areas with higher over-densities (Hoffman et al. 2012; Libeskind et al. 2015).

Two interesting and related studies recently reached apparently contradicting results. Alonso et al. (2015) reported that the halo mass function does not depend on the web-element type, but only on the local density, while Metuki et al. (2016) found the opposite. As suggested in the latter work, a possible explanation for this discrepancy is the fact that Alonso et al. (2015) defined the local density through a constant radius-smoothing kernel, while Metuki et al. (2016) used an adaptive kernel that explicitly depends on the virial radius of the haloes. The contradicting results of Alonso et al. (2015) and Metuki et al. (2016) reveal that the relation between environment and halo properties is still open to discussion and demands further investigation.

We have chosen a different approach to define the environment here that is based on a simple and clear-cut halo isolation criterion. We focus on haloes of a mass that would today host groups and clusters of galaxies. We chose to examine the behaviour of the halo abundance for isolated haloes within a specific radius, and similarly, of pairs of haloes, and compare it with the behaviour of less isolated haloes. The definition of environment in terms of isolation rather than in terms of specific values of the local density field or in terms of a web-element classification is also interesting from the observational point of view and can relatively easily be applied to redshift surveys.

\section{Simulation data}

We used halo catalogues of light-cone data generated on flight during the realization of a subset of N-body simulations from the "Dark Energy Universe Simulation" (DEUS) project (Alimi et al. 2010; Rasera et al. 2010; Courtin et al. 2011) that are publicly available through the DEUS database ${ }^{1}$. The N-body runs have been performed using the adaptive mesh refinement

\footnotetext{
1 wWw.deus-consortium . org/deus-data/
}

code RAMSES, which is based on a multigrid Poisson solver (Teyssier 2002; Guillet \& Teyssier 2011) for Gaussian initial conditions generated using the Zeldovich approximation with the MPGRAFIC code (Prunet et al. 2008) and input linear power spectrum from CAMB (Lewis et al. 2000). The light-cone data used here are from simulations of $2592 \mathrm{Mpch}^{-1}$ boxlength with $2048^{3}$ particles for a standard $\Lambda$ CDM model with parameters calibrated against supernova Type Ia from the UNION dataset (Kowalski et al. 2008) and measurements of the cosmic microwave background anisotropies from the Wilkinson Microwave Anisotropy Probe (WMAP) 5-year data (Komatsu et al. 2009), that is, $\Omega_{\mathrm{m}}=0.267$ and $H_{0}=100 \mathrm{~h} \mathrm{~km} \mathrm{~s}^{-1} \mathrm{Mpc}^{-1}$.

The light-cone halo catalogue covers the full sky out to a redshift $z<0.65$. The haloes contain more than 100 particles, while the particle mass resolution is $m_{\mathrm{p}}=1.5 \times 10^{11} M_{\odot} \mathrm{h}^{-1}$. Haloes were detected in the light-cone using the code $\mathrm{pFoF}$, a Friend-of-Friend halo finder (Roy et al. 2014). The total number of haloes in our catalogue is $\sim 3.15 \times 10^{6}$.

As a final note, we wish to stress that light-cone simulation data are extremely useful for testing algorithms and methodologies in order to enable direct comparisons with observational redshift data. Our particular simulation has the halo mass limit and volume traced, which makes it suitable for large statistical studies of the abundance and physical properties of massive haloes hosting groups and clusters of galaxies. This is indeed useful, since such objects will be studied in future cluster surveys, provided by ERosita (e.g. Hofmann et al. 2017) in the X-ray and LSST (e.g. LSST Science Collaboration et al. 2017) and EUCLID (e.g. Sartoris et al. 2016) in the optical.

\section{Method}

\subsection{Definition of local environment}

We here use a rather simple approach to define the local environment of dark matter haloes that is especially tailored to reveal the inter-halo dynamics. We avoid the approach of categorising the different regions of the cosmic web according to a range of web-elements (knots, filaments, sheets, and voids), and we use a criterion that is centred on each individual halo. In detail, we identified the nearest neighbour of each halo and its corresponding distance, which we call "isolation" radius, $R_{\text {isol }}$. Our criterion resembles that of Haas et al. (2012), although it is very different in that we defined the distance regardless of the halo mass $\left(M>10^{13} M_{\odot}\right)$.

The isolation radius defines a spherical region that is devoid of other haloes. Therefore, in the usual jargon, a very small isolation radius corresponds to a high-density region, while a large isolation radius corresponds to an underdense region (see the review by van de Weygaert et al. 2016). We note, however, that the true volume of such a "void" may be much larger as it may extend considerably towards directions other than that of the nearest neighbour. We also note that although our halo catalogue contains haloes with $M \geq 10^{13} M_{\odot}$, we used as central haloes (around which we defined the local environment) only those with $M \geq 2.5 \times 10^{13} M_{\odot}$. This is required in order to be able to define "isolation" towards lower mass haloes as well and thus ensure that our results are not heavily biased by the mass limit of our simulation halo data.

In Fig. 1, black points correspond to the frequency distribution of $R_{\text {isol }}$ for all haloes in the light-cone simulation, from which it is evident that the values of $R_{\text {isol }}$ span a wide range from $\sim 0.85$ to $37 \mathrm{~h}^{-1} \mathrm{Mpc}$. Evidently, the large majority of haloes have close neighbours, as expected from the hierarchical clustering scenario. 


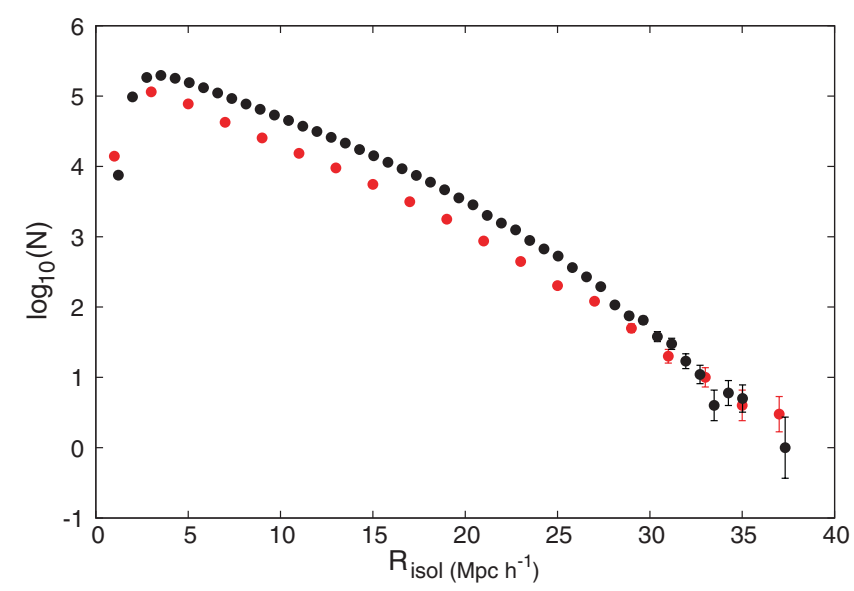

Fig. 1. Frequency distribution of the isolation radius: for the complete sample of light-cone DM haloes with $M \geq 2.5 \times 10^{13} M_{\odot}$ (black points) and for the sample of close pairs of DM haloes with separations $\leq 2.5 \mathrm{~h}^{-1}$ $\mathrm{Mpc}$ (red points). Error bars correspond to Poisson uncertainties.

However, the existence of extremely isolated haloes residing in huge underdense regions is particularly interesting; some of them are quite massive. We have found massive haloes $\left(M>10^{14} M_{\odot}\right)$ with isolation radii of up to $20-30 \mathrm{~h}^{-1} \mathrm{Mpc}$. This can give insight into halo formation processes in extreme environments.

Although haloes with small $R_{\text {isol }}$ should be considered as residing in high-density regions, we cannot exclude the possibility of isolated pairs of haloes. Selecting the central DM haloes with $R_{\text {isol }}<2.5 \mathrm{~h}^{-1} \mathrm{Mpc}$ and using the distance to the second nearest neighbour as a further isolation criterion, we find that there are many pairs of DM haloes (central halo plus its first neighbour) with different isolation status, some are even found within regions of radii as large as $\lesssim 38 \mathrm{~h}^{-1} \mathrm{Mpc}$ that are devoid of other haloes within the mass limit of our catalogue. In the same figure we show with red points the frequency distribution of the isolation radius for these close pairs of DM haloes, which also shows the wide range of isolation statuses of halo pairs.

A final but important methodological issue is related to the fact that by directly using the $R_{\text {isol }}$ parameter as a characterisation of local environment, we do not take the halo size into account, which unavoidably affects the available minimum separation among different halo sizes. Therefore, we chose in the remaining to use a parameterised characterisation of the local environment provided by the isolation radius in units of the halo virial radius, $R_{\text {isol }} / r_{\text {vir }}$. This definition is in accordance with the definition of local density introduced in Metuki et al. (2016), which is more reliable than the approach of Alonso et al. (2015), since the use of an adaptive radius smoothing kernel avoids the problem of underestimating the density around less massive haloes when the density is calculated at a fixed radius. Thus, the method of adaptive aperture is a way of normalising density with the virilization properties of each halo.

\subsection{Halo abundance function}

As described above, the main goal of this work is to study the environmental dependence of DM halo abundances using a simple but novel environmental isolation criterion. We note that we use the term "abundance function", $N_{\mathrm{AF}}(M)$, instead of "mass function" in order to highlight that we are applying our analysis on light-cone data, meaning that our catalogue has a wide span in redshift, in contrast to the "traditional" mass function, which is defined for specific redshifts. In order to quantify $N_{\mathrm{AF}}(M)$ for the different isolation status, it is essential to identify a versatile and relatively simple analytical function to fit the simulation halo abundances. Since the theoretically motivated $\Phi(M, z)$ 's, usually based on the Press \& Schechter formalism, reflect the whole population of haloes at a given redshift independent of their location, there is no direct way of applying them to the halo distribution in different environments unless one allows the numerical parameters to be fitted directly by the halo data in each different environment (see discussion relevant to Fig. 4). Moreover, our choice of selecting a simple and versatile analytical function was also made because we did not wish to make a detailed comparison of the great variety of theoretical $\Phi(M, z)$ in order to select an "optimum" model. Such a comparison has been performed in other studies (e.g. Watson et al. 2013) and is beyond the scope of the current work.

We thus identified a useful quantification of the DM halo abundances, based on a Schechter-like function, which is known to represent the luminosity function of galaxies accurately. The idea behind this choice is the expectation that a similar functional form can be expected to represent the DM halo abundances sufficiently well through the mass-to-light relation. A Schechter-like function, with a double power-law, was found to represent the abundances of our DM haloes accurately. We note that the second power-law, necessary to fit the high-mass end of the overall $\mathrm{AF}$, is not necessary when we consider DM haloes of medium and high isolation.

The functional form of the abundance function that we used is

$N_{\mathrm{AF}}(M)=\left[C_{1}\left(\frac{M}{M_{\star}}\right)^{\alpha}+C_{2}\left(\frac{M}{M_{\star}}\right)^{\beta}\right] \exp \left(-\frac{M}{M_{\star}}\right)$,

where $C_{1}$ and $C_{2}$ are normalisation factors related to the halo number density, $M_{\star}$ is the characteristic mass related to the knee of the abundance function, and $\alpha$ and $\beta$ are the exponents of the power laws.

The halo abundance as a function of mass was measured in logarithmic mass bins of width $\delta \log M \simeq 0.0693$, which is a compromise among the different subsamples that we used, in order that the halo numbers, in the most under-abundant bins, are not dominated by Poisson errors. The analytic fit to the resulting halo abundances was performed using the usual $\chi^{2}$ minimization procedure:

$\chi^{2}(\boldsymbol{p})=\sum_{i=1}^{N} \frac{\left(\log N_{i}(M)-\log N_{\mathrm{AF}}(M, \boldsymbol{p})\right)^{2}}{\sigma_{i}^{2}}$,

where $N_{i}$ is the number of haloes in the $i$ th mass bin, $\sigma_{i}$ is the uncertainty in $\log N_{i}$, for the calculation of which we chose to consider the uncertainty in $N_{i}$ equal to $\left.3 \sqrt{(} N_{i}\right)$. We note that we also used bootstrap uncertainties with no effect at all on our results. The sum is over the halo mass bins, and the vector $\boldsymbol{p}=\left(\alpha, \beta, M_{*}, C_{1}, C_{2}\right)$ contains the free parameters. The specific procedure that we used entails the following steps:

- We first fit the single power-law Schechter-like function and determined the best-fit values of $\alpha, C_{1}$ and $M_{\star}$.

- We then fit the double power-law Schechter-like function, but keeping the above three parameters constant to their bestfit values of the first step, that is, allowing only $C_{2}$ and $\beta$ to be fitted in this second step.

- If the reduced $\chi^{2}$ provided by the fit of the second step was lower than that of the first step, we considered that the double power-law version of the Schechter-like function is a better approximation to the halo abundance function under study. 


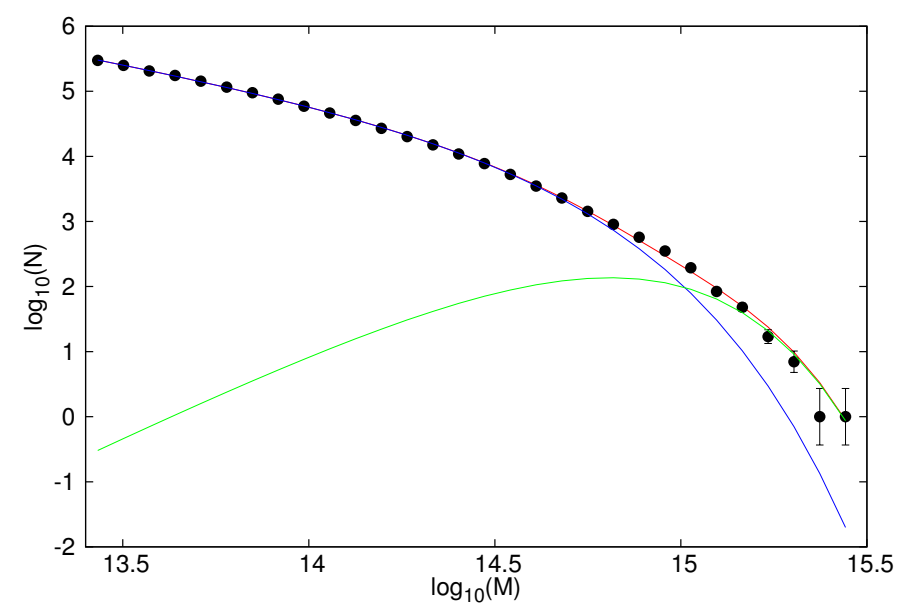

Fig. 2. Abundance of haloes with $M \geq 2.5 \times 10^{13} M_{\odot}$. The analytic Schechter-like function fits, $N_{\mathrm{AF}}(M)$, are represented by continuous curves. The single power-law Schechter function is shown in blue, the double power-law function in red, and in green we separately show the second power-law fit.

We also tested an alternative procedure by forcing the $C_{2}$ parameter to be fitted over a restricted range of low values, to take into account the small contribution of the corrective term, and then allowing all five free parameters to be fitted simultaneously, which led to results that were very similar to those of the previously described procedure, however.

As a manifestation of our procedure, we present in Fig. 2 the overall halo-abundance function of our complete light-cone halo sample (circular points) and the best-fit Schechter-like functions. The blue line corresponds to the single power-law fit (first step), where it is evident that it represents a wide dynamical range in mass well, except for the highest-mass regime. The green line corresponds to the second power-law fit, while the joint two powerlaw Schechter function of Eq. (1) is shown as the red curve. The excellent fit of the latter to the data is evident. We note that with our approach, the second Schechter-like function is used only as a small correction to the main fit, the parameters of which remain unchanged, meaning that the value of $M_{\star}$ that is used in the second Schechter-like function is fixed to the value determined by the fit to the initial single power-law Schechter function.

In order to investigate possible degeneracies among the parameters, we plot in Fig. 3 the $1 \sigma$ and $3 \sigma$ contours, corresponding to $\chi^{2}-\chi_{\min }^{2}=2.3$ and 11.83 , in the $\alpha$ and $M_{\star}$ solution space. Although there is an important degeneracy of the $\alpha$ parameter, the $M_{\star}$ parameter is very well constrained, with an extremely small uncertainty. This result is typical of all the different halo samples analysed in this work. The uncertainties of the individual parameters $\alpha$ and $M_{\star}$ are calculated after projecting the $1 \sigma$ surface on each parameter axis and estimating its maximum projected range. This definition provides a rather (artificially) wide uncertainty range. Alternatively, one could marginalize one parameter over the other and then estimate each individual parameter uncertainty, which would then, because of the significant degeneracy, provide an underestimate of the true uncertainties, however.

Finally, we tested the reliability of our results also with the Bayesian-based emcee (Foreman-Mackey et al. 2013) to estimate the best-fit values of the parameters of our model. emcee is an MIT-licensed Python implementation of the affine-invariant ensemble sampler for Markov chain Monte Carlo (MCMC) proposed by Goodman \& Weare (2010) and it is authored by Dan Foreman-Mackey. We found that both parameter estimation approaches give results that agree excellently well.

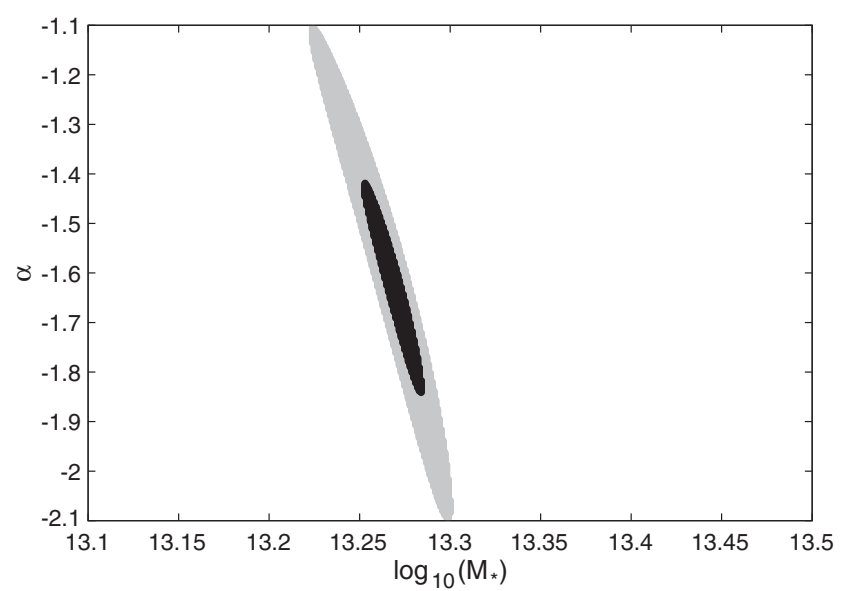

Fig. 3. $1 \sigma$ (black) and $3 \sigma$ (grey) contours in the $\alpha-M_{\star}$ parameter space.

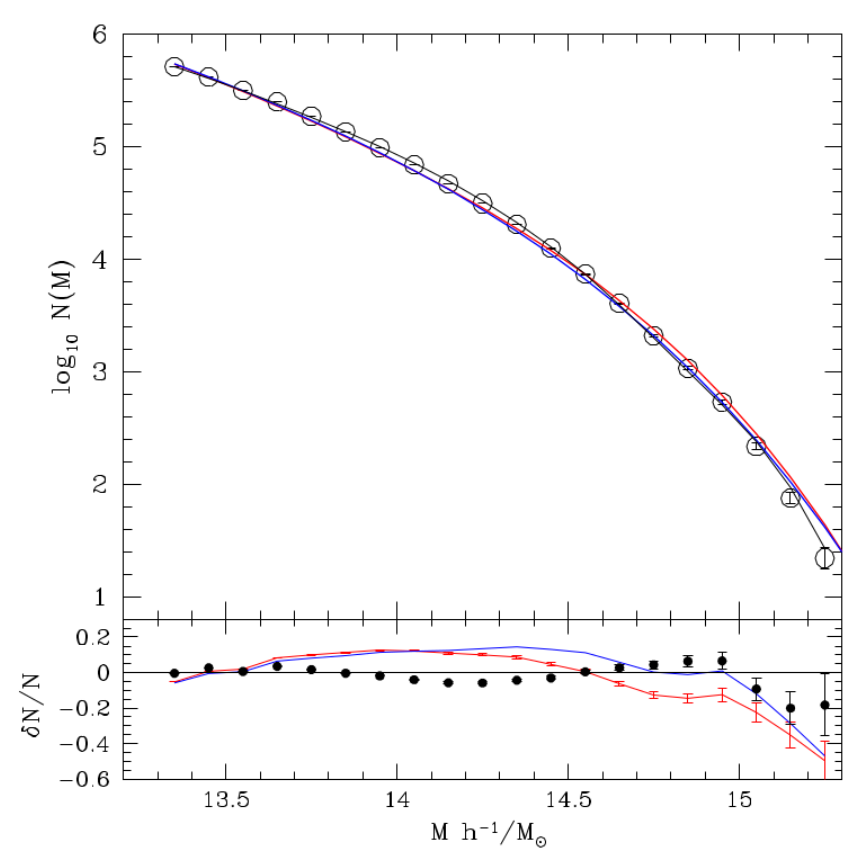

Fig. 4. Empty points represent the light-cone halo $N(M)$, the red curve shows the volume-integrated Reed et al. (2007) $\Phi(M, z)$, the blue line shows the Jenkins et al. (2001) $\Phi(M, z)$, and the black curve represents the double power-law Schecther function. The lower panel shows the relative deviations of the three models from the data $N(M)$, with the filled black points representing the deviation of our Schechter-like function. It is evident that the latter functional form represents the data better. The two theoretical $\Phi(M, z)$ are the best fits to the halo data from six models.

As a further test of our choice, we attempted to fit the abundance function of our light-cone haloes with various theoretical DM halo mass function models. To this end, we integrated over redshift the theoretically motivated $\Phi(M, z)$, within the volume of the light cone and found that the resulting theoretical abundance functions, $N \operatorname{th}(M)$, represent our data quite well. In Fig. 4 we present the two best-fit models to the halo data (from six models) and the double power-law Schechter form we used. The red curve in the figure corresponds to the volume-integrated Reed et al. (2007) model, the blue line to the Jenkins et al. (2001) model, and the black curve to the double power-law Schechter function. It is clear, especially from the lower panel, where we present the deviations of each model 
from the light-cone halo data, that the latter functional form is a much better fit to the data. This is also supported by the values of the reduced $\chi^{2}$, which for the theoretical models are more than an order of magnitude higher than for our fitting function.

We wish to add that we have also allowed the different numerical parameters of the theoretical mass functions to be fitted directly by the data, that is, we followed the same sort of approach as with our Schechter-like function, and although we find theoretical mass functions for which the obtained values of $\chi^{2} / \mathrm{df}$ are lower that than those obtained using their nominal parameter values, they are still significantly higher than the values corresponding to our Schechter fit.

Nevertheless, it is important to clarify that our aim in this work is not to introduce the Schechter-like form as an alternative to any theoretically motivated model, but only as a reliable quantification of the DM halo abundance function, which allows us to study its behaviour in different environments.

\section{Results}

In order to realize the main aim of our current work, we separated our halo catalogue into different subsamples based on their $R_{\text {isol }} / r_{\text {vir }}$ values. This allowed us to study the differences in the halo abundance function for haloes of different isolation status. The normalized AF, to the same overall number, for the two extreme cases of isolation status are shown in Fig. 5. The two AFs are significantly different, showing a strong dependence of the AF on the isolation status, with the halo AF of highly isolated halo regions being very steep and dominated by lower halo masses than in dense regions. For example, the most isolated haloes, corresponding to underdense regions, have masses that do not exceed $10^{13.75} M_{\odot}$, while haloes in dense regions span the entire mass interval. We also estimated the AF in all intermediate values of the halo isolation, and for each we fitted the function of Eq. (1), extracting the best-fit parameters $\alpha$ and $M_{\star}$. We find that $\alpha$ is a monotonically decreasing function of the isolation radius, taking values in the interval $\alpha \in[-5.2,-0.2]$, and the behaviour of $M_{\star}$, as a function of the isolation radius is shown in Fig. 6.

The generally decreasing tendency of both $M_{\star}$ and $\alpha$, up to values of $R_{\text {isol }} / r_{\text {vir }}<50$, is consistent with what we would expect; the denser the environment, the more massive the haloes that tend to form. However, we find an unexpected but statistically significant upturn of $M_{\star}$ for highly isolated haloes, implying that the most isolated haloes tend to be analogously more massive than in less extreme isolation cases.

In an attempt to understand this unexpected result, we investigated the behaviour of the characteristic mass $M_{\star}$ in two separate redshift bins to determine whether it shows signs of evolution. We performed our analysis separately in two redshift subsamples, for which we divided our total halo sample into two equal subsamples: one limited to $z<0.456$, and the other to $z \in(0.456,0.625)$.

As a first comparison of the haloes in the two redshift subsamples, we discuss the two extreme cases of environments; the densest $\left(R_{\text {isol }} / r_{\text {vir }}<4\right)$ and the most isolated $\left(50<R_{\text {isol }} / r_{\text {vir }}<\right.$ $70)$. We find that the lower redshift subsample contains only $\sim 10 \%$ of the total DM haloes with $R_{\text {isol }} / r_{\text {vir }} \in(50,70)$. We verified that this is not due to different comoving volumes probed by the two redshift intervals: the corresponding volumes are quite similar, $\sim 21.2 \mathrm{Gpc}^{3}$ and $\sim 27 \mathrm{Gpc}^{3}$ for the lower and higher redshift interval, respectively. A partial explanation of this difference would be if the DM haloes of the lower redshift subsample, as a result of further gravitational evolution, were to tend to have smaller virial radii. The mean virial radius in the

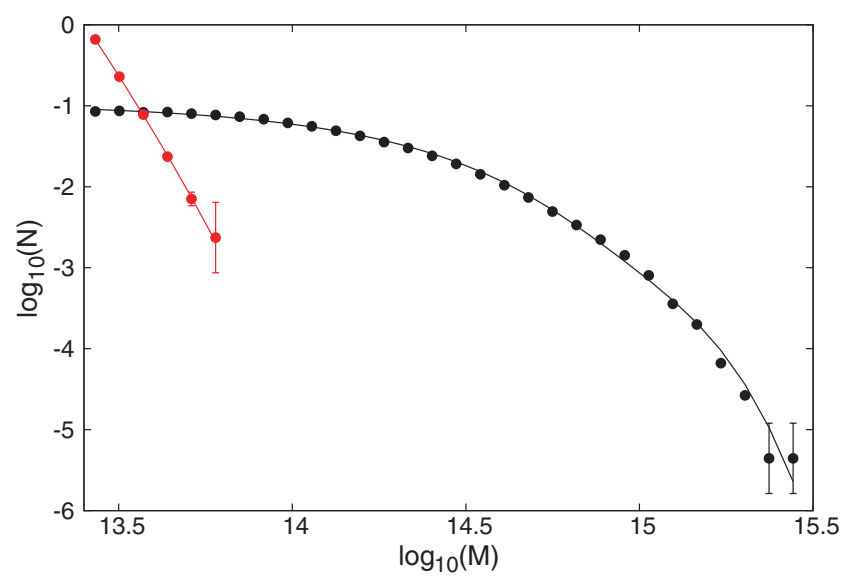

Fig. 5. Abundances of haloes of $R_{\text {isol }} / r_{\text {vir }} \leq 4$ (black) and $R_{\text {isol }} / r_{\text {vir }} \geq 50$ and best-fit curves. The AFs are normalized to the same total number.

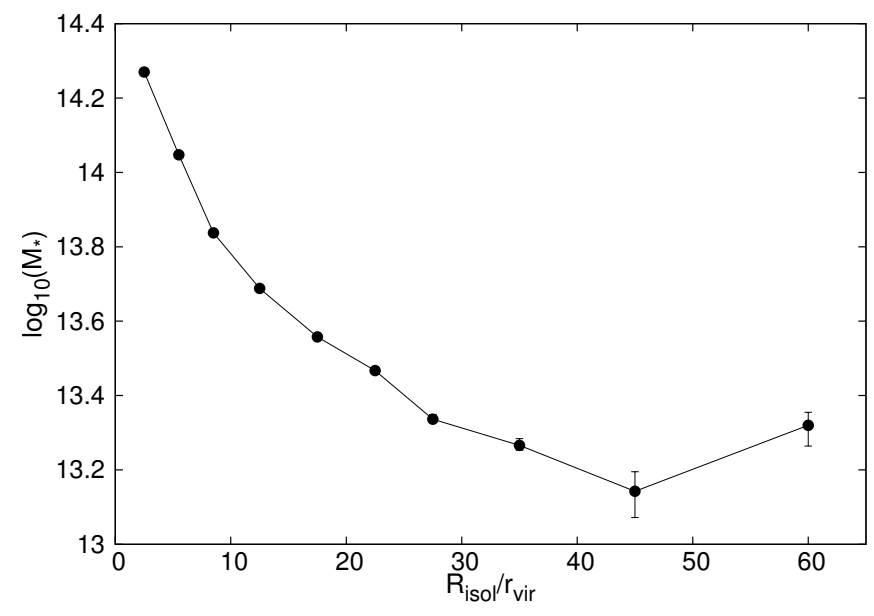

Fig. 6. Characteristic $M_{\star}$ parameter, with a significantly decreasing tendency except for the last bin, which corresponds to the most isolated haloes $\left(50<R_{\text {isol }} / r_{\text {vir }}<70\right)$.

two subsamples is indeed found to be $\left.\left\langle r_{\mathrm{vir}, 1}\right\rangle\right\rangle\left\langle r_{\mathrm{vir}, 2}\right\rangle$, while $\left\langle R_{\text {isol }, 1}\right\rangle \simeq\left\langle R_{\text {isol, } 2}\right\rangle$, resulting in lower values of $R_{\text {isol }} / r_{\text {vir }}$ in the low-redshift subsample. A further explanation, which appears to be supported by the results presented below, is that the largest isolation status of, especially, massive haloes evolves to smaller isolations at lower redshifts.

In Fig. 7 we compare the halo abundances for these two extreme cases of environment in the two different redshift intervals. Even though we have a relatively small number of highly isolated haloes and thus statistically important uncertainties, the normalized AF of the two different redshift intervals shows significant differences, some of which are expected. Specifically, we find that for the lowest isolation environment (high-density regions), the lower-redshift AF is systematically higher at the high-mass end than the higher-redshift AF, as expected from the gravitational growth of halo mass. However, at the highest isolations (lowest density regions), the opposite is true, which might be interpreted as meaning that the highest mass and most isolated haloes are more massive at higher $z$. This interpretation is rather unphysical and counter-intuitive, while an alternative, corroborated by the results presented below, is more probable.

The redshift-dependent differences were also quantified for all different isolation statuses by fitting the parameters of the abundance Schechter-like function as a function of $R_{\mathrm{isol}} / r_{\mathrm{vir}}$. 


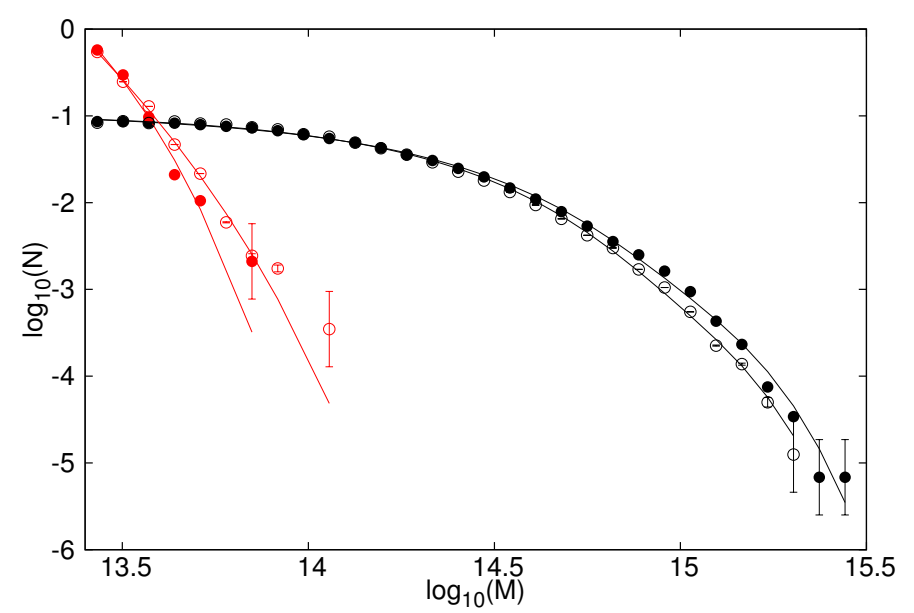

Fig. 7. Halo abundances for the two extreme different isolation status, $1<R_{\text {isol }} / r_{\text {vir }}<4$ (black) and $40<R_{\text {isol }} / r_{\text {vir }}<50$ (red). The lower redshift range $z<0.456$ is indicated with filled symbols, and the higher redshift range, $z \in(0.456,0.625)$, with open symbols. The AFs are normalized to the same total number.

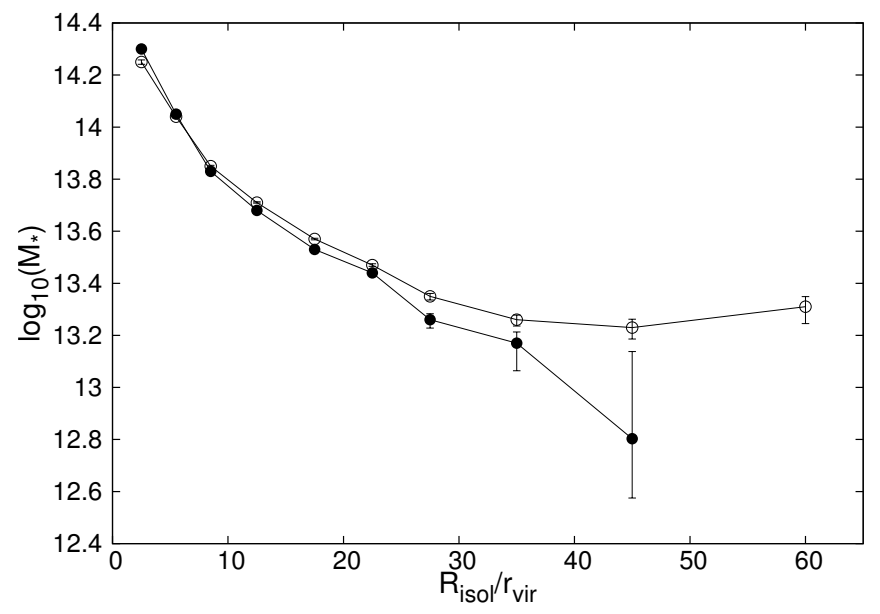

Fig. 8. Fitted $M_{\star}$ parameter of the Schechter-like AF as a function of $R_{\text {isol }} / r_{\text {vir }}$ for the two different redshift bins $(z<0.456)$ with filled symbols and $z \in(0.456,0.625)$ empty symbols. We observe an upturn only for $R_{\text {isol }} / r_{\text {vir }} \gtrsim 40$ only in the higher redshift bin.

The results of $M_{\star}$ for the two redshift intervals are presented in Fig. 8. $M_{\star}$ shows a consistent decrease with isolation status in both redshift intervals, except for the most isolated states, where the upturn in $M_{\star}$ is present only in the higher redshift bin. We note that the value of the $M_{\star}$ parameter denotes the position of the knee, that is, the mass above which the decreasing exponential term dominates. It is not necessary to have data over all mass scales to obtain the best-fit value of $M_{\star}$, not even scales corresponding to $M_{\star}$ itself. As we also explain below, in order to verify that the Schechter $N_{\mathrm{AF}}(M)$ fits the data well on such occasions, we tested some simple alternative forms to find that the Schechter function indeed fits the data better, even in cases when the best-fit value of $M_{\star}$ is lower than the mass limit of our catalogue.

Therefore, we verify that the statistical significant increase of $M_{\star}$ for the most isolated DM haloes, with respect to less isolated ones, is related to the higher redshift regime and that it disappears at lower redshifts. This finding, in full agreement with the results presented in Fig. 7 and discussed previously, implies that

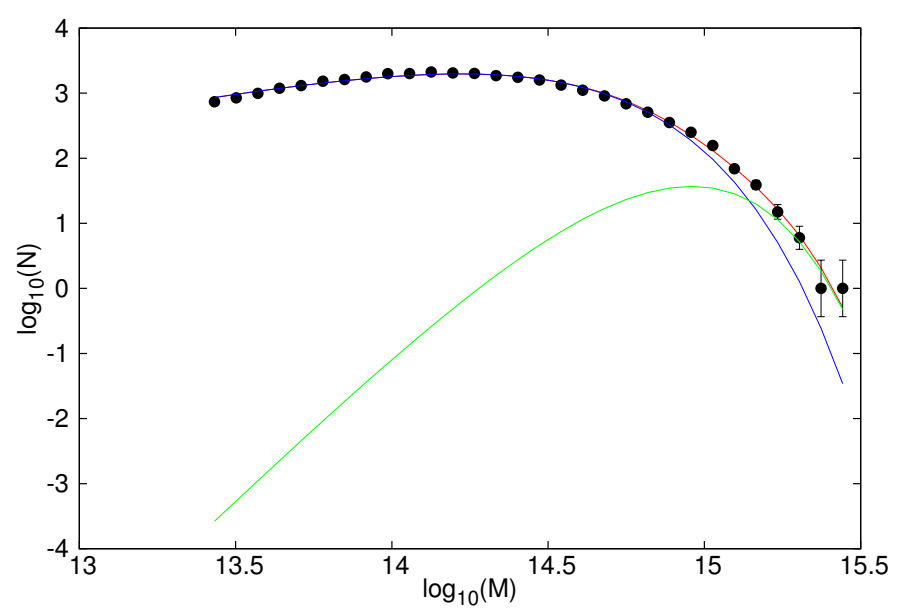

Fig. 9. AF of haloes that have a first and second nearest-neighbour with a distance $<4 r_{\text {vir }}$. The analytic Schechter-like function fits, $N_{\mathrm{AF}}(M)$, are represented by continuous curves. The single power-law Schechter function is shown in blue, the double power-law function in red, and in green we separately show the second power-law fit.

the large isolation of the most massive high- $z$ haloes, in other words, the large distance to their nearest neighbour, decreases with redshift. The specific mechanism that causes this behaviour is not clear and needs further study.

In order to scrutinize these results, especially in the light that the most isolated haloes span a relatively narrow range in halo mass and thus the range over which we can fit the analytical AF function is quite limited, which makes the resulting parameters of the Schechter-like function doubtful, we fit alternative functional AF forms to the data. In detail, we used a power law with two free parameters (the normalisation parameter, $C$, and the slope, $\alpha$ ) and an exponential with two free parameters (the normalisation parameter, $C$, and characteristic mass, $M_{\star}$ ). Comparing the resulting reduced minimum $\chi^{2}$ values, we found that our original Schechter form is the most suitable function to also represent the highly isolated halo AF.

Finally, we wish to compare our results with those of other studies that used more complex and multiparameter procedures to determine the environment (with all the advantages and disadvantages they may have): e.g. those of Metuki et al. (2016) and Alonso et al. (2015). Such a comparison should be made for the range of environments that can be identified as equivalent. These are the "knots", corresponding to the highest density semi-virialized cluster regions (e.g. Metuki et al. 2016). In our case, we constructed the AF for DM haloes with isolation radii of the first and second nearest neighbours (see Sect. 3.1) $<4 r_{\text {vir }}$. The AF we derived is shown in Fig. 9 and indeed has the characteristic downturn for masses $\lesssim 10^{14} M_{\odot}$, which is in qualitative agreement with the downturn described by Metuki et al. (2016) and Alonso et al. (2015) and also with what has been found from some of the web-element finders (T-web, V-web, and CLASSIC), which are compared in Libeskind et al. (2018) for knots (see their Fig. 6, top left panel). We note that the specific lower mass limit of our DM halo sample $\left(2.5 \times 10^{13} M_{\odot}\right)$ does not allow us to probe the observed upturn of the mass function towards lower halo masses described in Metuki et al. (2016) and Libeskind et al. (2018), a mass range where the latter works differ from Alonso et al. (2015). Moreover, a detailed comparison of our results with these works is not possible because the size of the simulation and its resolution are significantly different. 
Using the extended isolation criterion (that also includes the isolation of the second nearest neighbour and imposes that it lies within the same range as that of the first nearest neighbour) and repeating our main analysis of examining the behaviour of fitted parameters $\alpha$ and $M_{\star}$ as a function of isolation status, we found no significant change compared with our original analysis. The two free parameters have exactly the same tendencies, except for a systematic shift of the $\alpha$ parameter towards higher values, by 0.498 on average.

\section{Conclusion}

We studied the dependence of the halo abundances on environment of a light-cone $\Lambda \mathrm{CDM}$ halo catalogue extending to $z=0.65$, based on the DEUS simulation project. We used a distinct environmental criterion centred on each halo with $M>$ $2.5 \times 10^{13} M_{\odot}$ by defining an isolation region around it within which no other $M>10^{13} M_{\odot}$ halo can be found. A similar isolation criterion can easily be applied to observational data, which enables a direct comparison, with minimum assumptions, between simulations and observations. Our basic results are summarized below.

- The halo mass abundances depend strongly on the isolation radius, a result similar to that of many other studies that have defined the environment with a variety of multiparametric methods.

- A double power-law Schechter-like function fits the halo abundance of light-cone DM haloes for all isolation radii very well, although the second power-law is essential only for those of the lowest isolation status (highest density regions).

- The characteristic mass and the slope of the main powerlaw are decreasing functions of halo isolation, as expected from the gravitational growth of haloes in increasingly dense regions.

- An unexpected upturn of $M_{\star}$ occurs for the highest halo isolations, implying that the most isolated haloes tend to be analogously more massive than in less extreme isolation cases. This is present only at higher redshifts and disappears at lower redshifts, and it indicates an evolution of the isolation status of the most isolated relatively high-mass haloes towards lower isolations.

- The halo abundances of the less isolated haloes (i.e. those in the densest regions) show a downturn for $M \lesssim 10^{14} M_{\odot}$, in accordance with results related to "knots", based on various web-element finder algorithms.

We plan to use our isolation criterion to study the inter-halo dynamics in different environments, which might provide further insight into the structure and galaxy formation processes, in an attempt to investigate the extent to which the local environment is a dominant determining factor of physical processes.

Acknowledgements. PSC is supported by the European Research Council under the European Community's Seventh Framework Programme (FP7/2007-2013 Grant Agreement no. 279954). We also thank Kostas Karpouzas for his invaluable help in using the MCMC code.

\section{References}

Alimi, J.-M., Füzfa, A., Boucher, V., et al. 2010, MNRAS, 401, 775 Alonso, D., Eardley, E., \& Peacock, J. A. 2015, MNRAS, 447, 2683 Avila-Reese, V., Colín, P., Gottlöber, S., Firmani, C., \& Maulbetsch, C. 2005 ApJ, 634, 51

Bardeen, J. M., Bond, J. R., Kaiser, N., \& Szalay, A. S. 1986, ApJ, 304, 15

Blanton, M. R. \& Berlind, A. A. 2007, ApJ, 664, 791
Bond, J. R., Cole, S., Efstathiou, G., \& Kaiser, N. 1991, ApJ, 379, 440 Bond, J. R., Kofman, L., \& Pogosyan, D. 1996, Nature, 380, 603 Boselli, A. \& Gavazzi, G. 2006, PASP, 118, 517 Bullock, J. S., Kolatt, T. S., Sigad, Y., et al. 2001, MNRAS, 321, 559 Corasaniti, P. S. \& Achitouv, I. 2011, Phys. Rev. Lett., 106, 241302 Courtin, J., Rasera, Y., Alimi, J.-M., et al. 2011, MNRAS, 410, 1911 Croton, D. J., Gao, L., \& White, S. D. M. 2007, MNRAS, 374, 1303 Doroshkevich, A. G. 1970, Astrophysics, 6, 320

Dressler, A. 1980, ApJ, 236, 351

Eardley, E., Peacock, J. A., McNaught-Roberts, T., et al. 2015, MNRAS, 448, 3665

Foreman-Mackey, D., Hogg, D. W., Lang, D., \& Goodman, J. 2013, PASP, 125, 306

Forero-Romero, J. E., Hoffman, Y., Yepes, G., et al. 2011, MNRAS, 417, 1434 Gao, L. \& White, S. D. M. 2007, MNRAS, 377, L5

Gao, L., White, S. D. M., Jenkins, A., Stoehr, F., \& Springel, V. 2004, MNRAS, 355,819

Gao, L., Springel, V., \& White, S. D. M. 2005, MNRAS, 363, L66

Gómez, P. L., Nichol, R. C., Miller, C. J., et al. 2003, ApJ, 584, 210

Goodman, J. \& Weare, J. 2010, Comm. App. Math. Comp. Sci., 5, 65

Guillet, T. \& Teyssier, R. 2011, J. Comput. Phys., 230, 4756

Haas, M. R., Schaye, J., \& Jeeson-Daniel, A. 2012, MNRAS, 419, 2133

Hahn, O., Carollo, C. M., Porciani, C., \& Dekel, A. 2007, MNRAS, 381, 41

Harker, G., Cole, S., Helly, J., Frenk, C., \& Jenkins, A. 2006, MNRAS, 367, 1039

Hoffman, Y., Metuki, O., Yepes, G., et al. 2012, MNRAS, 425, 2049

Hofmann, F., Sanders, J. S., Clerc, N., et al. 2017, A\&A, 606, A118

Jenkins, A., Frenk, C. S., White, S. D. M., et al. 2001, MNRAS, 321, 372

Kaiser, N. 1984, ApJ, 284, L9

Komatsu, E., Dunkley, J., Nolta, M. R., et al. 2009, ApJS, 180, 330

Kowalski, M., Rubin, D., Aldering, G., et al. 2008, ApJ, 686, 749

Lee, C. T., Primack, J. R., Behroozi, P., et al. 2017, MNRAS, 466, 3834

Lemson, G. \& Kauffmann, G. 1999, MNRAS, 302, 111

Lewis, A., Challinor, A., \& Lasenby, A. 2000, ApJ, 538, 473

Libeskind, N. I., Knebe, A., Hoffman, Y., et al. 2011, MNRAS, 411, 1525

Libeskind, N. I., Hoffman, Y., Knebe, A., et al. 2012, MNRAS, 421, L137

Libeskind, N. I., Hoffman, Y., Forero-Romero, J., et al. 2013, MNRAS, 428, 2489

Libeskind, N. I., Knebe, A., Hoffman, Y., \& Gottlöber, S. 2014, MNRAS, 443, 1274

Libeskind, N. I., Tempel, E., Hoffman, Y., Tully, R. B., \& Courtois, H. 2015, MNRAS, 453, L108

Libeskind, N. I., van de Weygaert, R., Cautun, M., et al. 2018, MNRAS, 473, 1195

LSST Science Collaboration (Marshall, P., et al.) 2017, ArXiv e-prints [arXiv: 1708.04058]

Ma, C.-P., Maggiore, M., Riotto, A., \& Zhang, J. 2011, MNRAS, 411, 2644

Martínez, H. J. \& Muriel, H. 2006, MNRAS, 370, 1003

Maulbetsch, C., Avila-Reese, V., Colín, P., et al. 2007, ApJ, 654, 53

Metuki, O., Libeskind, N. I., Hoffman, Y., Crain, R. A., \& Theuns, T. 2015, MNRAS, 446, 1458

Metuki, O., Libeskind, N. I., \& Hoffman, Y. 2016, MNRAS, 460, 297

Muldrew, S. I., Croton, D. J., Skibba, R. A., et al. 2012, MNRAS, 419, 2670

Navarro, J. F., Frenk, C. S., \& White, S. D. M. 1997, ApJ, 490, 493

Peacock, J. A. \& Heavens, A. F. 1985, MNRAS, 217, 805

Plionis, M. \& Basilakos, S. 2002, MNRAS, 329, L47

Press, W. H. \& Schechter, P. 1974, ApJ, 187, 425

Prunet, S., Pichon, C., Aubert, D., et al. 2008, ApJS, 178, 179

Ragone-Figueroa, C. \& Plionis, M. 2007, MNRAS, 377, 1785

Rasera, Y., Alimi, J.-M., Courtin, J., et al. 2010, AIP Conf. Ser., 1241, 1134

Reed, D. S., Bower, R., Frenk, C. S., Jenkins, A., \& Theuns, T. 2007, MNRAS, 374, 2

Roy, F., Bouillot, V. R., \& Rasera, Y. 2014, A\&A, 564, A13

Sartoris, B., Biviano, A., Fedeli, C., et al. 2016, MNRAS, 459, 1764

Schuecker, P., Böhringer, H., Reiprich, T. H., \& Feretti, L. 2001, A\&A, 378, 408

Sheth, R. K. \& Tormen, G. 1999, MNRAS, 308, 119

Sheth, R. K. \& Tormen, G. 2004, MNRAS, 350, 1385

Sheth, R. K., Mo, H. J., \& Tormen, G. 2001, MNRAS, 323, 1

Teyssier, R. 2002, A\&A, 385, 337

Tinker, J. L., Robertson, B. E., Kravtsov, A. V., et al. 2010, ApJ, 724, 878

Valageas, P. 2009, A\&A, 508, 93

van de Weygaert, R., Shandarin, S., Saar, E., \& Einasto, J. 2016, IAU Symp., 308

Warren, M. S., Abazajian, K., Holz, D. E., \& Teodoro, L. 2006, ApJ, 646, 881

Watson, W. A., Iliev, I. T., D'Aloisio, A., et al. 2013, MNRAS, 433, 1230

Wechsler, R. H., Bullock, J. S., Primack, J. R., Kravtsov, A. V., \& Dekel, A. 2002, ApJ, 568, 52

Wechsler, R. H., Zentner, A. R., Bullock, J. S., Kravtsov, A. V., \& Allgood, B. 2006, ApJ, 652, 71

Zhu, G., Zheng, Z., Lin, W. P., et al. 2006, ApJ, 639, L5 